\title{
Dinámica de las exportaciones de carne bovina de Brasil y de Uruguay: un análisis comparativo
}

\section{Dynamics of beef cattle exports from Brazil and Uruguay: a comparative analysis}

\author{
Mariana R.Espalter de Moraes* y João Garibaldi Almeida Viana
}

Universidade Federal do Pampa.Santana do Livramento, Rio Grande do Sul, Brasil.

( ${ }^{\star} E$-mail: marianaespalter@gmail.com)

http://dx.doi.org/10.19084/RCA14115

Recebido/received: 2014.08.14

Recebido em versão revista/received in revised form: 2015.08.02

Aceite/accepted: 2015.08 .03

\section{Resumen}

Brasil es uno de los principales países exportadores de carne bovina del mundo, juntamente con Uruguay, país vecino con grandes potenciales en la producción del sector bovino y por eso uno de los mayores competidores de Brasil. Debido a la importancia del sector bovino en ambos países, este trabajo tiene por objetivo analizar la evolución de las exportaciones de carne bovina de Brasil y de Uruguay a lo largo del período 2001-2013, verificando el volumen y el precio de las exportaciones en dicho período. El análisis estadístico siguió el Método Clásico Multiplicativo de Series Temporales. Se evidenció una tendencia ascendente del volumen total exportado de carne bovina tanto para Brasil cuanto para Uruguay. De la misma forma, los precios se presentaron crecientes, el precio de la carne bovina exportada por Uruguay presentó un crecimiento anual del 11,28\% más elevado que Brasil con un crecimiento de 9,96\% al año. Ambos países presentaron exportaciones estacionales, habiendo una expansión en el período de zafra y retracción del volumen en el período de entre zafra. En un análisis de largo plazo, se constató que los ciclos de exportación no están bien determinados, imposibilitando trazar patrones futuros.

Palabras-llave: economía agrícola, economía internacional, exportaciones, ganadería.

\begin{abstract}
A bstract
Brazil is one of the major exporters of beef in the world, together with Uruguay, neighbor country with great potential in the production of the beef sector and therefore one of the major competitors with Brazil. Because of the importance of the beef sector in both countries, this paper aims to analyze the evolution of beef exports from Brazil and Uruguay over the period 2001-2013, checking the volume and price of exports in that period. The statistical analysis followed the Classical Multiplicative Time Series Method. An upward trend of total exports of beef to both Brazil to Uruguay as was evidenced. Likewise, prices had increased; the price of beef exported by Uruguay showed an annual growth of $11.28 \%$, higher than Brazil with a growth of $9.96 \%$ year. Both countries had seasonal exports, with an expansion in the production period and volume shrinkage during the period between produce. In a long-term analysis, it was found that the export cycles are not well determined, making it impossible to draw future patterns.
\end{abstract}

Keywords: agricultural economics, beef cattle, exports, international economics.

\section{INTRODUCCIÓN}

La cadena productiva de la carne bovina es un sector importante del agro negocio brasileño y uruguayo. La conexión de los agentes y la búsqueda por la eficiencia en la producción pecuaria se inició con la constitución de complejos agroindustriales. El Complejo Agroindustrial (CAI) puede ser definido, según Müller (1982), como: “[...] conjunto de los procesos técnico-económicos y socio políticos que envuelven la producción agrícola, el beneficio y su transformación, la producción de bienes industriales para la agricultura y los servicios financieros y comerciales correspondientes [...]."

En Brasil, durante la década de 1970, ocurrió un 
proceso de modernización agrícola que culminó en los complejos agroindustriales, aumentando la utilización de nuevos insumos agropecuarios y proporcionando aumento de la productividad. Con la implementación de este modelo, se buscó alinear la agricultura en el desarrollo económico, a través de la oferta de capital y de la generación y difusión de tecnologías, intensificando así, el aumento de la producción de alimentos y materias primas (Paiva, 1979).

Posteriormente, a mediados de los años 1990, las exportaciones de carne bovina presentaron un incentivo en Brasil con la implantación del Plan Real. El plan económico tenía por objetivo estabilizar la economía al controlar la inflación, generando también un aumento del consumo interno y de la competitividad entre los sectores del país. También se observó un crecimiento de las exportaciones bovinas debido al perfeccionamiento del sector frente a las cadenas cárnicas competidoras, como por ejemplo, el sector avícola y porcino (Brandão, 2007). Así, en el período entre 1997 y 2002 hubo un crecimiento de $193 \%$ del volumen de exportaciones de carne bovina en el país, convirtiendo a Brasil en el principal exportador mundial del producto (Neves et al., 2003).

A su vez, el complejo agroindustrial de carne bovina de Uruguay, a partir de la década del 1990, interrumpió un ciclo de estancamiento, a través de la calidad del producto y la rigidez sanitaria, dirigiendo el sector para la exportación, atendiendo las diversas exigencias del mercado externo (Mondelli y Zylbersztajn, 2008). Esas mudanzas transformaron a Uruguay en protagonista en el mercado internacional, posicionándose como uno de los principales exportadores de carne bovina y ovina y potencial competidor de Brasil en el comercio de carnes.

A partir de la importancia del mercado externo para el sector bovino, ¿cuál fue la dinámica de las exportaciones de carne bovina de las dos regiones? En este contexto, el objetivo del artículo fue analizar la evolución de las exportaciones de carne bovina de Brasil y de Uruguay en el período de 2001-2013, dando especial atención: al volumen comercializado, al precio del producto y a los tipos de carne exportados por los países en ese período.

Con los resultados comparativos, se busca trazar limitaciones y/o potencialidades de la estructura exportadora del sector bovino de Brasil y de
Uruguay, contribuyendo a la comprensión del mercado externo de los dos países. Además, la investigación tiene por finalidad auxiliar a los agentes de la cadena productiva en el proceso de la toma de decisiones, por medio de la comprensión de las relaciones de comercio y posibilidades de crecimiento.

El artículo está ordenado en cinco secciones. Después de la introducción, el tópico de material y métodos es presentado, delineando la metodología necesaria para lograr el objetivo propuesto. Los resultados y discusión presentan el producto de los análisis econométricos y relacionan las evidencias del caso empírico con la literatura especializada. Luego enseguida, las conclusiones sintetizan los principales resultados encontrados, delimitando las principales limitaciones de la pesquisa y sus potencialidades para futuros trabajos. Al final, se presenta las referencias bibliográficas utilizadas en la investigación.

\section{MATERIAL Y MÉTODOS}

El estudio adoptó el método cuantitativo de investigación, por medio del análisis de series temporales. Los procedimientos partieron de la colecta de datos secundarios mensuales, extraídos del Sistema Alice Web del Ministerio de Desarrollo, Industria y Comercio Exterior (MDIC, 2013) y del banco de datos del Instituto Nacional de Carnes del Uruguay (INAC-Uruguay). El análisis estadístico de los datos se basó en el método clásico multiplicativo de series temporales para análisis de las exportaciones (Morettin y Tolo, 1987; Pindyck y Rubinfeld, 2005). La elección por el método multiplicativo se justifica por la posibilidad de descomposición de la serie en tendencia, utilizándose la unidad variable (cantidad exportada), y los demás componentes, estacionalidad y ciclos, valiéndose de índices, obteniendo como valor 1 (uno) la media de la serie en el periodo.

$$
Y_{i}=T_{i} \times S_{i} \times C_{i} \times I_{i}
$$

Donde: $\mathrm{Ti}$ = tendencia de largo plazo en la serie; $\mathrm{Si}=$ componente estacional; $\mathrm{Ci}=$ componente cíclico de largo plazo; $\mathrm{Ii}=$ componente irregular o residual.

Inicialmente, los datos fueron sometidos al análisis de estacionalidad, por medio del método de 
ajuste estacional (Pindyck y Rubinfeld, 2005) en la obtención de índices estacionales correspondientes a cada mes del año. Los índices estacionales reflejan las fluctuaciones periódicas relativamente regulares que ocurren dentro de cada periodo de 12 meses, año tras años (Levine et al., 2011). Con el cálculo de los índices se buscó analizar las oscilaciones de corto plazo del volumen y precio de las exportaciones en el período 2001 a 2013.

Después, los datos temporales de volumen y precio de las exportaciones fueron organizados para el análisis del componente de tendencia, a partir de la armonización por medio del cálculo de medias móviles centradas de 12 períodos (ecuación 2) a fin de extraer el componente estacional e irregular de la serie.

$$
\tilde{y}_{t}=\frac{1}{12}\left(y_{t+6}+\cdots+y_{t}+y_{t-1}+\cdots+y_{t-5}\right)
$$

Para el análisis de tendencia de largo plazo se utilizó el método de extrapolación simple que está basado en el desarrollo de un modelo de regresión determinante de series temporales de una única ecuación, denominado tendencia lineal. La tendencia del volumen y precio de las exportaciones fue estimada por medio de un modelo de regresión lineal (ecuación 3) y regresión semilogarítmica (ecuación 4) para análisis de la tasa de variación mensual.

$$
\begin{aligned}
& Y_{t}=\alpha+\beta t+\varepsilon \\
& \ln Y_{t}=\alpha+\beta t+\varepsilon
\end{aligned}
$$

Donde: $Y t=$ volumen y precio de carne bovina exportada; $\ln Y i=$ logaritmo natural del volumen y precio exportado; $\alpha=$ intercepto; $\beta=$ coeficiente angular; $t=$ tiempo de la serie histórica representado por los meses del año; $\varepsilon \mathrm{i}=$ residuo.

El análisis de la tendencia buscó verificar un patrón general o persistente de largo plazo, ascendente o descendente (Levine et al., 2011). La existencia o no de tendencia declinante o ascendente fue verificada a través del test de hipótesis t-student a un nivel máximo de $5 \%$ de significancia.

En la regresión semilog, la interpretación de los coeficientes fue hecha en forma de tasa de variación mensual, conforme a la ecuación 5, basada en la denotación de Wooldridge (2005), lo que posibilitó la comparación del comportamiento del volumen y del precio de las exportaciones brasileñas y uruguayas.

$$
\% \Delta Y_{t} \approx(100 \cdot \beta) \Delta t
$$

De acuerdo a lo anterior, se determinó el componente cíclico de los volúmenes de carne, para ambos países y los diferentes tipos, por medio de la división de la serie armonizada por la estimación de la tendencia conforme a la ecuación 6 .

$$
C_{i}=\frac{M M_{i}}{Y_{t}}
$$

Donde: $\mathrm{Ci}=$ componente cíclico; $\mathrm{MMi}=$ media móvil centrada mensual; $\mathrm{Yt}=$ valor mensual de tendencia para la variable.

Los ciclos reflejan repetidas oscilaciones o movimientos ascendentes o descendientes a lo largo de cuatro fases: pico, contracción (recesión), fondo (depresión) y expansión (recuperación) (Levine et al., 2011). El cálculo del componente cíclico permitió analizar la variación de los volúmenes de carne a largo plazo, identificando periodos de alta y baja exportación y la existencia de regularidades del comportamiento de las exportaciones de carne bovina.

Por lo tanto, el método clásico de series temporales permitió el análisis del volumen y del precio de las exportaciones del sector bovino brasileño y uruguayo, para auxiliar la comparación de las trayectorias del sector de ambos países.

\section{RESULTADOS Y DISCUSIÓN}

La ganadería es una actividad pecuaria que se destaca tanto en la economía brasileña como en la uruguaya. Por ser un sector representativo cuyos países poseen ventaja comparativa, el excedente de producción es destinado a la exportación. En el ranking mundial el mayor exportador de carne bovina es Brasil, ya Uruguay ocupa la octava posición en el ranking, de acuerdo con los datos de 2005 a 2009 (Borges y Mezzadri, 2009). La figura 1 representa la evolución de las exportaciones de carne bovina de Brasil y de Uruguay de 2001 a 2013, representada por la cantidad total de carne bovina exportada, en kilogramos.

Se observa, por el comportamiento del volumen de las exportaciones mensuales, una característica 


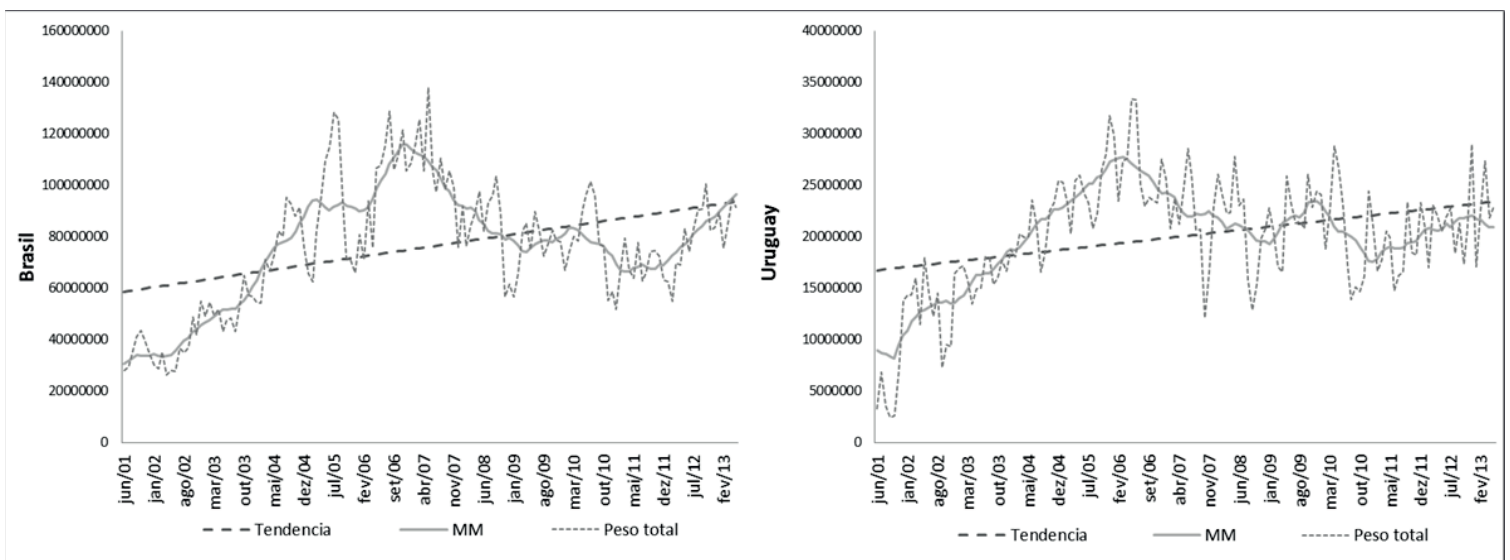

Figura 1 - Tendencia, medias móviles (MM) y cantidad (kilogramos) de carne bovina brasileña y uruguaya exportada en el periodo de junio de 2001 a mayo de 2013.

estacional y cíclica de las exportaciones de carne bovina en el periodo, corroborada por patrón de la serie de volumen amenizada (medias móviles), indicando fluctuaciones de largo plazo bien determinadas. Sin embargo, a pesar de las oscilaciones de corto y largo plazo se visualiza una tendencia de crecimiento del volumen de carne bovina exportada de 2001 a 2013, puntualizando una expansión de las exportaciones de carne brasileña y uruguaya en el mercado internacional.

Al analizar la figura, se percibe que hasta mediados del año 2006 hay un crecimiento continuo y significativo del volumen de las exportaciones de carne bovina en los dos países. No obstante, después de este periodo, las exportaciones pasan a sufrir una retracción hasta el año 2010, volviendo a expandirse a partir de 2011. Para Pereira (2009), uno de los factores responsables por el aumento significativo de las exportaciones brasileñas a partir de 2001, fue el brote de fiebre aftosa ocurrido en Argentina, uno de los principales competidores del sector. Conforme al autor, Brasil expandió sus exportaciones ocupando parte del mercado externo argentino.

Entre los años 2006 y 2007 se constató un aumento en el número de faena de hembras del sector bovino en Brasil, del orden del 48,1\%. Debido a una caída del precio del producto y una elevación del costo de producción, reduciendo la reproducción de los animales y, en consecuencia de eso, reduciendo también la oferta interna de carne bovina. Otro motivo que puede haber influenciado la caída de las exportaciones de carne bovina originadas en Brasil, está relacionado a la caída del consumo mundial, debido a la crisis mundial financiera de
2008; donde el mayor importador de carne bovina brasileña, Rusia, disminuyó en aproximadamente $50 \%$ sus importaciones de carne bovina. Otro factor importante en el mercado internacional es la tasa de cambio que en 2009 presentó una valorización del real frente al dólar, disminuyendo la competitividad de Brasil en el mercado externo, generando una contracción en las exportaciones de carne (Borges y Mezzadri, 2009).

Como factores determinantes en las variaciones de las exportaciones de Uruguay en ese período, Montes (2008) destaca el aumento significativo del stock de cabezas de bovinos en el país de 2001 a 2004, estabilizándose en 2005 y contrayéndose entre los años 2006 y 2007. Así, a partir del 2007 se verifica una caída del número de faenas relacionada a la sustitución de grandes áreas de engorde por la agricultura, causando escasez de pasturas para la pecuaria, además de una fuerte sequía en Uruguay registrada en el año 2006. Después, en 2008 se verificó un aumento poco significativo $(1,7 \%)$ del stock de rebaño bovino uruguayo (Montes, 2008).

De esta forma, la variación de stock interno y su rigidez de oferta a partir de 2006, son factores que se relacionan con la caída de las exportaciones de carne bovina uruguaya iniciada en el mismo año. Sin embargo, se visualiza un desequilibrio acentuado en el volumen de exportaciones de carne bovina uruguaya entre los años 2009 y 2010, desequilibrio este que fue influenciado por factores climáticos, o sea, la sequia entre los años 2008 y 2009 que perjudico la crianza del rebaño bovino para la exportación, haciendo que el precio de la carne bovina aumentase y disminuyese la demanda por el producto (Montes, 2009). Adicionalmente, la crisis financiera 
mundial también impacto en el mercado de carnes, generando una caída en las exportaciones bovinas originadas en el Uruguay en el año 2009.

La tabla 1 representa los coeficientes de la estimación de los modelos de tendencia lineal y semilogarítmica para las exportaciones mensuales de bovinos de 2001 a 2013. A partir del análisis de regresión se puede cuantificar el comportamiento de tendencia de la exportación de carne bovina de Brasil y de Uruguay. El volumen de exportación de carne bovina originado en Brasil puede ser analizado tanto en su totalidad como por los tipos: congelada y resfriada. Pero el volumen de carne bovina uruguaya puede ser analizado apenas en su totalidad exportada.

Se nota que los coeficientes angulares de los modelos se mostraron significativos para los dos tipos se evidencia una tasa media mensual de variación de las exportaciones de carne bovina resfriada de $-0,31 \%$ en el periodo, o sea, un decrecimiento anual de las exportaciones de carne bovina resfriada en el orden de $-3,72 \%$ de 2001 a 2013.

En el año del 2007 la Unión Europea, el mayor importador de carne bovina, realizó un embargo a la carne bovina brasileña, impidiendo la entrada del producto en su territorio debido a no corresponder con sus exigencias sanitarias, de calidad y trazabilidad (Oliveira et al., 2012). Este hecho, juntamente con otras ocurrencias, puede haber influenciado la caída de las exportaciones de carne bovina brasileña de tipo resfriada en el año 2008. Dado que este tipo de carne es más perecedero y demanda un mayor cuidado con las exigencias, así como, la carne resfriada es la más demandada por los mercados más exigentes, siendo impuestas

Tabla 1 - Coeficientes de regresión lineal y semilogaritmica de tendencia para la exportación de carne bovina (Kg) de Brasil (resfriada, congelada y volumen total) y de Uruguay (volumen total)

\begin{tabular}{l|c|c|c|c}
\hline Modelo & Intercepto $(\boldsymbol{\alpha})$ & Coef. angular $(\boldsymbol{\beta})$ & $\boldsymbol{t}$ calculado & Valor $\boldsymbol{p}$-fisher \\
\hline Brasil resfriada & & & & \\
Yt & $12.028 .425,98$ & $-30.561,06$ & $-4,95$ & 0,00 \\
Ln Yt & 16,2664 & $-0,0031$ & $-4,65$ & 0,00 \\
\hline Brasil congelada & & & & \\
Yt & $46.419 .976,08$ & $275.306,30$ & 7,59 & 0,00 \\
Ln Yt & 17,5163 & 0,0058 & 9,33 & 0,00 \\
\hline Brasil total & & & & \\
Yt & $58.448 .402,07$ & $244.745,25$ & 6,44 & 0,00 \\
Ln Yt & 17,7766 & 0,0044 & 7,99 & 0,00 \\
\hline Uruguay total & & & & \\
Yt & $16.705 .895,41$ & $46.511,40$ & 5,89 & 0,00 \\
Ln Yt & 9,6497 & 0,0031 & 6,99 & 0,00 \\
\hline
\end{tabular}

Fuente: estimado por los autores.

de carne brasileña $(\mathrm{P}<0,01)$, resfriada y congelada; así como para el volumen total de exportaciones de ambos países. Estos resultados indican el rechazo de la hipótesis nula, es decir, la existencia de un patrón ascendente de tendencia de exportación total, tanto brasileña cuanto uruguaya en la última década, y un patrón de caída en el volumen de exportaciones brasileñas de carne bovina del tipo resfriada. Para la exportación de carne bovina brasileña, de tipo resfriada, a partir de los datos de estimación de tendencia lineal, se observa un decrecimiento de $30.561,06 \mathrm{Kg}$ de carne bovina resfriada exportada a cada variación de un mes en el periodo. En base a la estimación semilogarítmica, cuotas de importación impidiendo el acceso y la competencia del producto brasileño (Silva et al., 2010).

A su vez, el mercado de exportación de carne bovina del tipo congelada se presenta en expansión. En base a los datos de la estimación lineal, se constata un crecimiento medio de $275.306,30$ quilogramos de carne bovina congelada exportada a cada variación mensual del periodo. En este sentido, se destaca el crecimiento de las exportaciones de carne bovina congelada en los últimos doce años. En la interpretación semilogarítmica, se observa una tasa de variación mensual positiva de $0,58 \%$ 
determinando un crecimiento anual medio en las exportaciones de carne bovina brasileña congelada de $6,96 \%$ en el período analizado.

La carne bovina del tipo congelada es menos perecedera cuando es comparada a la carne de tipo resfriada. La durabilidad de la carne congelada es de hasta 2 años, mientras que la carne resfriada dura serie de exportaciones de la pecuaria de corte bovina presenta un comportamiento zafral acentuado. A partir del cálculo de índices de estacionalidad fue posible medir la variación estacional de la exportación de carne bovina en los dos países, o sea, las oscilaciones de corto plazo, en el periodo de 12 meses, conforme demuestra la figura 2.

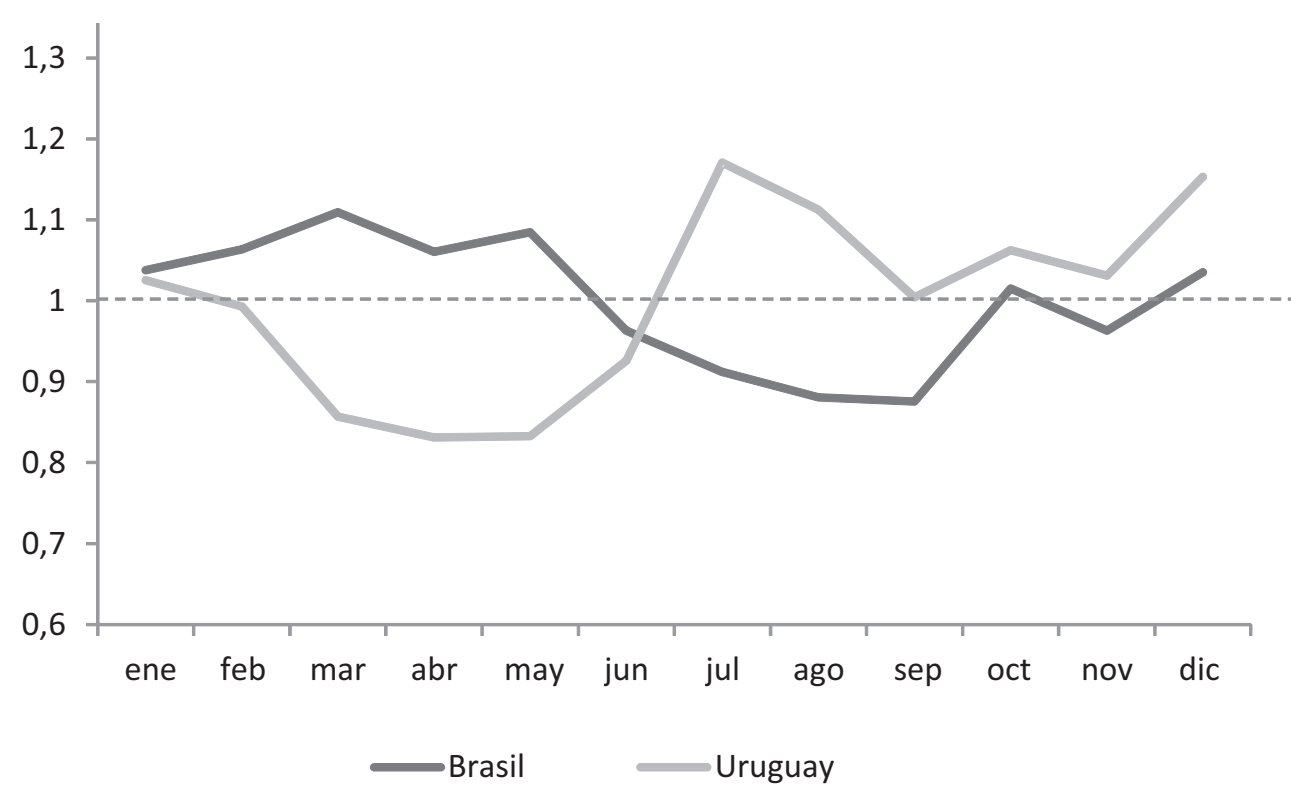

Figura 2 - Índices de estacionalidad de las exportaciones totales de carne bovina en el periodo de 2001 a 2013 - comparativo entre los países.

cerca de 120 días (Silva et al., 2010). Debido al mayor tiempo de conservación de la carne congelada, es posible afirmar que es mayor la facilidad de acceso a los mercados de mayor distancia física y por eso su volumen es más elevado en comparación al volumen de carne del tipo resfriada. La cuestión de menor complejidad en el transporte de este tipo de carne también es un factor favorable a su comercialización en el exterior.

En el año 2010 se constató en Brasil la exportación de 80.696 toneladas de carne bovina resfriada y 870.559 toneladas de carne bovina del tipo congelada, pudiéndose así verificar la gran diferencia entre los volúmenes debido a las exigencias y cuidados con cada tipo de carne, como lo dicho anteriormente. Al visualizar el volumen total de exportaciones, tanto de Brasil como de Uruguay, se percibe un aumento anual significativo de las exportaciones de carne bovina de 5,28\% y 3,72\%, respectivamente, a lo largo de los años 2011 al 2013. Aliado a un patrón de tendencia de largo plazo, la
En el análisis de los índices, el valor 1 se refiere a la media de las exportaciones del periodo analizado. Fluctuaciones a lo largo de los meses indican un comportamiento superior o inferior a la media de la serie, determinando expansión o retracción del mercado internacional de cada país. Tal análisis, puede ser hecho de forma porcentual al observar la distancia de cada índice mensual en relación al valor 1 (media del periodo) y multiplicar por cien.

Al comparar los índices de estacionalidad entre los dos países en el periodo de 2001 a 2013 se notan comportamientos estacionales contrarios hasta el mes de Setiembre. Al visualizar la figura se puede percibir que el principal período de contracción de la exportación de carne bovina del Brasil fue entre mes de julio y septiembre, con una caída de aproximadamente $15 \%$ en relación a la media. Ya para Uruguay, se percibe que los meses de marzo y mayo presentaron comportamiento semejantes, con una restricción de las exportaciones de 15\% debajo de la media. Los valores mensuales de los 
índices de estacionalidad de las exportaciones de los dos países son presentados en la Tabla 2.

El comportamiento estacional en la exportación de carne bovina en Brasil es explicado por cuestiones internas de zafra y entre zafra, así como por el periodo de cría (estación de monta). El período de zafra del sector bovino está marcado por el primer semestre del año, donde ocurren lluvias con mayor frecuencia, desarrollando pasturas para la alimentación del ganado. Pero el periodo de entre zafra, está marcado por el segundo semestre del año, en los meses de invierno, donde las pasturas son escasas debido a causas climáticas como el frio (DEPEC, 2012) y escasez de lluvia en regiones del centro y del norte. Por lo tanto se nota una restricción de las exportaciones de carne bovina brasileñas durante los meses de otoño e invierno debido al período de entre zafra y, una expansión de las exportaciones en el periodo de zafra.

En una perspectiva de largo plazo, la figura 3 presenta el componente cíclico de las exportaciones de carne bovina de Brasil y de Uruguay de 2001 a 2013. Los ciclos de producción determinan las variaciones en el volumen de exportación que tienden a repetirse a lo largo del tiempo. El índice cíclico representado por el valor 1 (uno) indica la tendencia del volumen de exportaciones en el período, posibilitando identificar restricción y expansión de las exportaciones en el largo plazo a partir de índices inferiores y superiores, respectivamente.

Se confirma en los ciclos de exportación del sector bovino brasileño una variación regular, con un patrón cíclico marcado por alta y caída de las exportaciones. Las cuatro fases del comportamiento cíclico (pico, contracción, depresión y expansión)

Tabla 2 - Índices de estacionalidade de las exportaciones de carne bovina de Brasil yde Uruguay en el periodo de 2001 a 2013

\begin{tabular}{l|c|c}
\hline Mes & Brasil & Uruguay \\
\hline Enero & 1,038 & 1,026 \\
Febrero & 1,064 & 0,993 \\
Marzo & 1,109 & 0,857 \\
Abril & 1,060 & 0,831 \\
Mayo & 1,085 & 0,833 \\
Junio & 0,963 & 0,926 \\
Julio & 0,912 & 1,171 \\
Agosto & 0,880 & 1,112 \\
Septiembre & 0,876 & 1,005 \\
Octubre & 1,015 & 1,063 \\
Noviembre & 0,963 & 1,031 \\
Diciembre & 1,035 & 1,153 \\
\hline
\end{tabular}

Fuente: estimado por los autores.

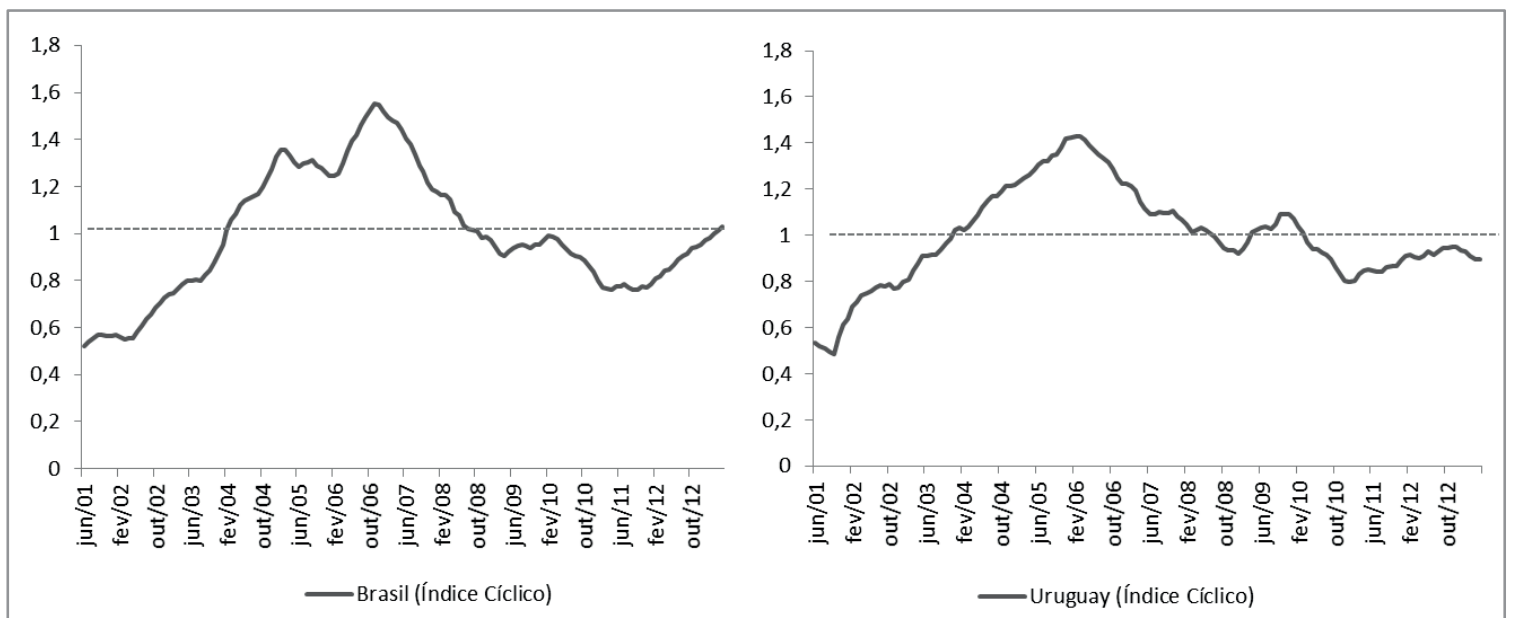

Figura 3 - Ciclos de la cantidade de carne bovina brasileña y uruguaya exportada en el período de 2001 a 2013. 
no se presentan bien definidas. Las fases de pico y depresión alcanzan una variación en las exportaciones en relación a la tendencia de exportación del período, de aproximadamente $60 \%$ por encima de la media y 50\% debajo de la media, respectivamente. Además de eso, con el análisis de los ciclos, se nota que el mercado de exportaciones bovinas brasileñas está entrando en un período de expansión, o sea, exportaciones por encima de la línea de tendencia de elevación de las exportaciones del período de 2001 a 2013.

La caída de las exportaciones a partir de 2007 está relacionada a cuestiones internas y externas del país. Como fue mencionado anteriormente, entre los años de 2006 y 2007 hubo un aumento significativo en las faenas de hembras bovinas en Brasil, perjudicando el rumbo de la oferta del producto. A partir de 2008, con la crisis mundial, los países pasan a sufrir las consecuencias de la misma, reduciendo el consumo y restringiendo sus flujos comerciales. En 2009 una valorización del real frente al dólar perjudicó el flujo de las exportaciones de carne bovina brasileña.

Al visualizarse la figura 3, referente a los ciclos de la exportación de carne bovina uruguaya en el período de 2001 a 2013, se aprecia que no hay una existencia de ciclos bien determinados, y por tanto no es posible verificar la existencia de las cuatro fases del ciclo (pico, contracción, depresión y expansión). Se constata la existencia de oscilaciones a largo y a corto plazo, con pequeñas caídas y picos, característicos de desequilibrios tanto internos como externos del país.

Las fases de pico y depresión alcanzan una variación en el volumen de las exportaciones de Uruguay del $40 \%$ encima de la media y 50\% debajo de la media, respectivamente, en relación a la tendencia de exportación del período. Además de eso, se aprecia que las exportaciones uruguayas están entrando en una fase de estabilidad, siguiendo la línea de tendencia ascendente, de acuerdo con la figura 1. Entre los años de 2003 y 2005 la expansión significativa del volumen de carne bovina exportada por Uruguay ocurrió debido a los buenos precios de Estados Unidos por carne congelada para ser utilizada en la industria manufacturera (Mondelli y Zylbersztajn, 2008).

Un factor importante a ser considerado en este análisis es el precio de las exportaciones de carne bovina, originadas en Brasil y en Uruguay. Se utilizan los precios en dólares por tonelada de carne bovina comercializada, para mejor comprensión y comparación entre los países. De acuerdo con la figura se puede visualizar la tendencia, medias móviles y la relación precio en dólares por tonelada de las exportaciones de carne bovina brasileña y uruguaya a lo largo del período de 2001 a 2013.

Al analizar el comportamiento mensual del precio de las exportaciones de carne bovina brasileña y uruguaya, se nota que hay una tendencia creciente de los precios a lo largo del período, corroborada por la recta de tendencia ascendente. Se puede visualizar, por medio de la serie armonizada (medias móviles) que en el año 2007 y entre los años 2009 y 2010, los precios tuvieron un aumento significativo, inclinando aún más la recta de la tendencia. Es posible afirmar que el aumento significativo del precio de la carne bovina brasileña exportada esté relacionado a la contracción del volumen

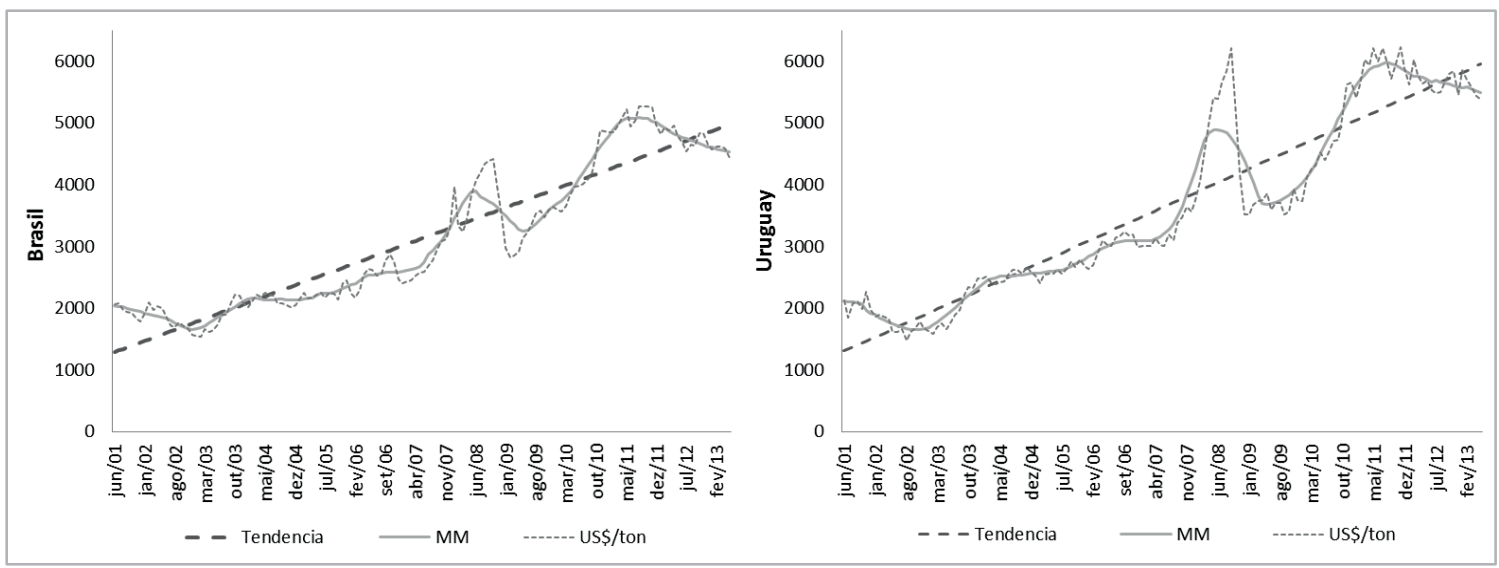

Figura 4 - Tendencia, Medias Móviles (MM) y precio (US\$/tonelada) de carne bovina brasileña y uruguaya exportada en el período de junio de 2001 a mayo de 2013. 
exportado en el mismo período. Tal contracción se dio por cuestiones internas de producción en Brasil, debido al descarte de hembras ocurrido en 2006, reduciendo la oferta y, consecuentemente, las exportaciones. Debido a la escasez de oferta, el precio del producto presentó una suba. En el año 2008 se visualiza una caída significativa del precio, debido a la crisis mundial, donde el consumo fue reducido induciendo a un exceso de la oferta.

Se aprecia un comportamiento de los precios de las exportaciones de carne bovina uruguaya semejante a la variación de precios del mercado brasileño. Se verifica una tendencia ascendente de los precios, con elevaciones acentuadas en los años 2007 y 2010, entre los años de 2008 y 2009 se verifica una caída acentuada del precio, así como en los precios brasileños. Los motivos de tales aumentos en los precios pueden estar relacionados a la escasez de oferta del producto, debido al período de sequía sufrido por Uruguay en el año 2006 y posteriormente en 2008 y 2009. Debido a los factores climáticos, hubo una caída en las faenas de bovinos y en consecuencia de eso, una caída en las exportaciones del producto. Otro factor importante a ser destacado es la sustitución de la ganadería por el plantío de granos, ocurrido a mediados de 2007 en Uruguay, reduciendo los rebaños bovinos. La caída de los precios verificada entre los años 2008 y 2009 son reflejos de la crisis mundial, debido a la caída del consumo y restricciones en el comercio internacional, generando un excedente de producción.

Un factor importante a ser destacado es el énfasis mundial en la producción y consumo de biocombustibles (etanol y biodiesel) sustituyendo en parte el consumo de combustibles fósiles. Los biocombustibles son derivados de cereales, tales como: maíz, azúcar y semillas oleaginosas, dichos cereales necesitan áreas cultivables para ser producidos (FAO, 2007). Sin embargo, hay preocupación con respecto a la sustitución de áreas que antes cultivaban alimentos y que pasaron a cultivar cereales para la producción de biocombustibles (Bermann, 2008). Por estos motivos hubo una retracción mundial de la oferta de carne bovina, debido a la sustitución de las áreas, pero en contrapartida, el aumento del consumo de productos derivados de proteína animal se continuó expandiendo debido al aumento de la renta de los países emergentes. Este escenario de retracción de la oferta y el aumento de la demanda resultó en un aumento de precios de la carne bovina en el mercado internacional, como se puede ver en la figura 4 .
Se destaca, al comparar la evolución de precio de ambos países, que el precio de las exportaciones de carne bovina uruguaya crece a un ritmo más acelerado que el precio de las exportaciones brasileñas. Los coeficientes angulares de los modelos de precio se muestran significativos $(P<0,01)$ en todos los casos, tanto para el precio del volumen total cuanto para el precio de los tipos de carne separadamente. En el caso del tipo de carne brasileña resfriada, esta es más valorizada en relación al tipo de carne congelada. De acuerdo con la estimación de tendencia hay un aumento mensual de US\$ 34,50 por tonelada de carne resfriada brasileña a lo largo del período analizado. Con base en la estimación semilogarítmica, se confirma una tasa media mensual de variación de precio de las exportaciones de carne bovina resfriada de $0,79 \%$ por mes, o sea, un aumento anual del precio de las exportaciones de carne bovina brasileña (contabilizadas en toneladas) de $9,48 \%$ a lo largo del período.

De acuerdo con lo que fue destacado anteriormente en cuanto al volumen de exportaciones de carne bovina, del tipo resfriada, vale recordar que este tipo de carne tiene acceso a países más exigentes. Por ser una carne con menor tiempo para consumo, demanda mayores cuidados tanto en la conservación cuanto en el transporte hasta llegar al país destinado. Todos esos factores hacen que el precio sea más elevado. Además, Brasil debe atender la demanda y exigencias de sus consumidores, lo que también encarece el producto y requiere tiempo para adaptar la estructura productiva. De la misma forma, el precio por tonelada de carne bovina, de tipo congelada, presenta una expansión, y en mayor grado cuando se compara con el tipo de carne resfriada. A través del cálculo de tendencia se puede afirmar que hay un aumento mensual de US\$26,10 por tonelada de carne bovina congelada para exportación, a lo largo de los años 2001 al 2013. De acuerdo con los datos obtenidos a través de la estimación semilogarítmica se verifica una tasa media mensual de variación de precio de las exportaciones de carne bovina brasileña, de tipo congelada, de $0,93 \%$ al mes, o sea, hay un aumento de precio por tonelada de carne bovina brasileña de tipo congelada, de 11,16\% al año durante el período.

Comparando el precio por tonelada del volumen total de exportación de carne bovina de Brasil y de Uruguay, se advierte que hay un aumento más acentuado del precio de la carne uruguaya a lo largo del período. La carne brasileña presenta un aumento anual del precio por tonelada del orden de 
9,96\%; mientras que la carne bovina uruguaya para exportación presenta un aumento anual de precio del orden de $11,28 \%$. De forma comparativa, en el mes de octubre del año 2013, según el último precio disponible y recogido, se verifica la diferencia de precio por tonelada de carne bovina para exportación entre Brasil y Uruguay. El precio brasileño cerró en US\$ 4.548,01 y el precio uruguayo cerró en US\$ 5.304,00. Se deduce que el producto de origen uruguayo posee un precio $16,6 \%$ más elevado que el producto brasileño.

Esa diferenciación de precio puede estar asociada a la calidad de la carne en los diferentes países, incluyendo factores que envuelven desde las razas del rebaño hasta mismo la alimentación y el tipo de crianza de los animales. Así como también la adaptación y acompañamiento de los mercados de acuerdo con las exigencias de los consumidores, por ejemplo, cuestiones de sanidad y trazabilidad que influencian en el precio y en la calidad del producto.

Uruguay por ser un país de economía pequeña y abierta, para destacarse en el mercado internacional frente a los competidores, necesita diferenciar su producto y agregar valor al mismo, para de ese modo, poder insertarse en el mercado competitivo. De esta forma, el país atiende las exigencias de sus consumidores, como por ejemplo, América del Norte y Europa, a través de las exigencias higiénicas sanitarias y de trazabilidad. Tales estrategias de inclusión en el mercado internacional traen un aumento en la calidad del producto y un aumento en los costos, a consecuencia de eso el precio se eleva (Mondelli y Zylbersztajn, 2008).

Según la afirmación de Mondelli y Zylbersztajn (2008) "por un lado el precio internacional de la carne bovina aumentó, pero por otro la condición sanitaria mejor de Uruguay permitió ampliar los mercados, mejorando el mix de los negocios de exportación de carne bovina y el precio medio de exportación"; lo que corrobora los resultados encontrados en ese estudio. Uruguay exporta por año, $75 \%$ de su producción bovina, accediendo a los más exigentes mercados consumidores de carne bovina: Estados Unidos, Canadá y Unión Europea (Mondelli y Zylbersztajn, 2008). Por otro lado, la cadena productiva brasileña ha presentado evoluciones significativas, debido al mejoramiento genético y a la sanidad, así como inversiones en la producción y en la gestión. Brasil es uno de los países que posee menores costos de producción del sector bovino, siendo en su mayoría producción a pasto, debido a su ventaja comparativa en poseer grandes extensiones de tierra. La crianza a pasto favorece el status del producto afuera del país, por estar relacionado a la menor incidencia de enfermedades, como por ejemplo, la Encefalitis Espongiforme Bovina (BSE), más conocida como la enfermedad de la vaca loca, y también por relacionarse con el bien estar animal (Pereira, 2009).

\section{CONCLUSIONES}

Se identificó una tendencia de aumento del volumen total exportado por Brasil a lo largo del período de 2001 a 2013 así como, se verificó una tendencia ascendente de los precios (US\$/tonelada) del volumen total durante el mismo período. De la misma forma, se puede constatar, por medio del análisis de series temporales, una tendencia creciente del volumen total de carne bovina exportada por Uruguay durante el mismo período, acompañada por la también creciente tendencia de los precios (US\$/tonelada).

En cuanto a las cuestiones zafrales, se vio variaciones en el volumen total de las exportaciones a lo largo de los meses. En el caso brasileño los meses que presentan pico y expansión de las exportaciones son marzo, mayo y diciembre, representados por un volumen de $10 \%$ por encima de la media. Para Uruguay, la expansión de las exportaciones de carne bovina ocurre en los meses de julio y diciembre, con un volumen de $20 \%$ por encima de la media. El análisis de largo plazo está corroborado por el índice cíclico, donde se puede ver que tanto para Brasil cuanto para Uruguay no hay existencia de ciclos bien determinados del volumen de exportaciones de carne bovina. Para Uruguay las fases de pico y depresión alcanzan una variación en el volumen de las exportaciones de $40 \%$ por encima de la media y $50 \%$ por debajo de la misma, respectivamente, en relación a la tendencia de exportación del período. Las exportaciones uruguayas están entrando en una fase de estabilidad. Para Brasil las fases de pico y depresión alcanzan una variación en las exportaciones, en relación a la tendencia de exportación del período de aproximadamente $60 \%$ por encima de la media y $50 \%$ por debajo de la misma, respectivamente, y se puede constatar que las exportaciones brasileñas están entrando en una fase de expansión. 
Como principales limitaciones del análisis se puede destacar la indisponibilidad de datos secundarios finales de exportaciones para el año 2014, lo que posibilitaría un análisis más recenté del comportamiento del mercado internacional de carne bovina, así como la falta de un análisis más específico con respeto a los principales mercados importadores y sus influencias en los precios de la commodity de los dos países estudiados. Como desafío para futuras investigaciones, se destaca la potencialidad de la estimación de los modelos econométricos para previsión de las exportaciones de carne bovina, sumado a la posibilidad del análisis de la cointegración de las series.

\section{REFERENCIAS}

Bermann, C (2008) - Crise ambiental e as energias renováveis. Ciência e Cultura, vol. 60, n. 3, p. 20-29.

Borges, A.R. y Mezzadri, F.P. (2009) - Bovinocultura de corte. Análise da Conjuntura Agropecuária, safra 2009/2010. Estado do Paraná, Secretaria da Agricultura e do Abastecimento, Departamento de Economia Rural.

Brandão, F.T.; Ferreira Junior, J.C.; Brichi, L.O. y Miranda, ITP. (2007) - Exportação da carne bovina nacional: os desafios que o setor enfrentará nos próximos anos frente às novas exigências do mercado internacional. Maringá Management: Revista de Ciências Empresariais, vol. 4, n. 2, p. 7-14.

DEPEC (2012) - Agronegócio Brasil em foco. Fevereiro 2012. Departamento de Pesquisa e Estudos Econômicos; Bradesco. [cit. 3-2-2014]. http://www.economiaemdia.com.br/EconomiaEmDia/ pdf/AgronegocioBrasilemFoco_27-02-12.pdf.

FAO (2007) - Food and Agriculture Organization of the United Nations [cit. 21-2-2014].

http://www.fao.org/newsroom/es/news/2007/ 1000620/index.html.

Graner, F. (2006) - Superávit da balança comercial do agronegócio é de US 38,4bi. O Estado de São Paulo.Comércio Exterior. [cit. 3-2-2014]. http://acervo.estadao.com.br/pagina/\#!/ 20060106-40988-nac-29-eco-b14-not.

Levine, D.M.; Stephan, D.F.; Krehbiel, T.C. y Berenson, M.L. (2011) - Estatística: teoria e aplicações. Rio de Janeiro, LTC.

MDIC (2013) - Sistema de Análise das Informações de Comércio Exterior - ALICEWEB, 2013. < http://aliceweb2.mdic.gov.br/>
Mondelli, M. y Zylbersztajn, D. (2008) Determinantes dos arranjos contratuais:o caso da transação produtor-processador de carne bovina no Uruguai. Revista de Economia e Sociologia Rural, vol. 46, n. 3, p. 831-868.

http://dx.doi.org/10.1590/S0103-20032008000300010

Montes, E.N. (2008) - ¿Un quiebre de tendencia en el stock vacuno? Proyección al 30 de junio de 2008. Revista Plan Agropecuario, vol. 126, p. 32-35.

Montes, E.N. (2009) - La ganadería bovina del Uruguay del siglo XXI. Revista Plan Agropecuario, vol. 131, p. 58-62.

Morettin, P.A. \& Tolo, C.M. (1987) - Séries temporais. São Paulo, Atual.

Müller, G. (1982) - Agricultura e industrialização do campo no Brasil. Revista de Economia Política, vol. 2, n. 6, p. 47-77.

Neves, M.F; Scare, R.F. y Cavalcanti, M.R. (2003) Comercialização internacional de produtos animais provenientes de pastagens: a Carne Bovina. In: Anais do $20^{\circ}$ Simpósio Sobre Manejo de Pastagem "Produção Animal em Pastagens - Situação Atual e Perspectivas". Realizado em Piracicaba- SP, 4 de Setembro de 2003. ISBN: 85-7133-021-2. [cit. 10-9-2013].

http://www.beefpoint.com.br/cadeia-produtiva/ especiais/comercializacao-internacional-deprodutos-animais-provenientes-de-pastagens-7237/.

Oliveira, L.C.; Rocha, A. y Piveta, T. R. (2012) - A carne bovina como alternativa para aumento das exportações brasileiras para a União Européia: análise sobre a carne bovina. In:III Fateclog Congresso de Logística das Faculdades de Tecnologia do CEETEPS. Guaratingueta.

Paiva, R.M. (1979) - Teoria da modernização agrícola:uma apreciação crítica. In: Paiva, R.M. (Ed.) A agricultura no desenvolvimento econômico: suas limitações como fator dinâmico. Brasília, IPEA.

Pereira,P.R.R.X.(2009)-Rastreabilidadeesanidade:desafios para as exportações brasileiras de carne bovina. Tese Mestrado em Agronegócios, Universidade Federal do Rio Grande do Sul, Porto Alegre.

Pindyck, D.L. \& Rubinfeld, R.S. (2005) - Econometria: modelos e previsões. Rio de Janeiro, Campus.

Silva, R.F.; Caixeta-Filho, J.V. y Zucchi, J.D. (2010) A logística da carne bovina: produtos desossados e refrigerados. Agroanalysis, vol. 30, p. 36-37.

Souza, F.P. (2008) - O Mercado da Carne Bovina no Brasil.Revista Académica: Ciências Agrárias e Ambientais, vol. 6, n. 3, p. 427-434.

Wooldridge, J. (2005) - Introdução à econometria: uma abordagem moderna. São Paulo, Thomson Learning. 